\title{
Design and Implementation of Media Player Based on Android
}

\author{
Maoqiang Song, Jie Sun, Xiangling Fu, Wenkuo Xiong \\ School of Software Engineering \\ Beijing University of Posts and Communications \\ Beijing, China \\ Sunjie0324@gmail.com
}

\begin{abstract}
As a new open-source smart mobile phone platform, Android has become one of the popular cell phone systems. Many users like to watch Video by a mobile phone, but the original media player in Android has many limits. For example, the media formats supported are few and the decode efficiency is fair. First, the paper designs a new media player system structure based on hiberarchy. Then, the paper designs the decode module based on FFmpeg decode library and the display module based on SDL. At last, the paper describes the implementation of the new media player in Android.
\end{abstract}

Keywords-Android; media player; FFmpeg; SDL

\section{INTRODUCTION}

The original meaning of the word "android" is robot, here Android is a open-source cell phone platform which is released by OHA(Open Handset Alliance) on November 12, 2007 [1][2].The platform is the first integrate and open-source mobile software platform, which includes operating system, middleware, user interface and core applications. Many developers paid close attention to Android from the very beginning, just because its openness made it easy to handle for the developers who belong to third party. Because of its openness, easy-to-use, free and easy-to-develop, Android platform has a bright future. Today the function of a mobile phone is powerful, playing a video on a mobile phone becomes a basic function, but the original media player in Android can't meet people's need since there're various formats of media files, so a powerful media player based on Android is needed.

The decode library is the most complex part of a media player, it will be a huge project if we develop it by ourselves. At present, the decode module of most media players is based on FFmpeg decode library, such as Strom, KMP Player, TCPMP, etc. The FFmpeg [3] is a set of open-source and cross-platform libraries for multimedia applications. FFmpeg decode library supports over 90 formats of media files, such as AVI, MPEG, OGG, $3 \mathrm{GP}$, etc. In this paper we also design the decode module based on FFmpeg decode library.

This paper designs and implements a powerful media player based on Android which supports most media formats.

\section{ANDROID ARCHITECTURE}

The architecture of Android consists of five main components from the top down: Application, Application Framework, Libraries, Android Runtime and Linux Kernel as shown in Fig. 1 as follows [4] [5].

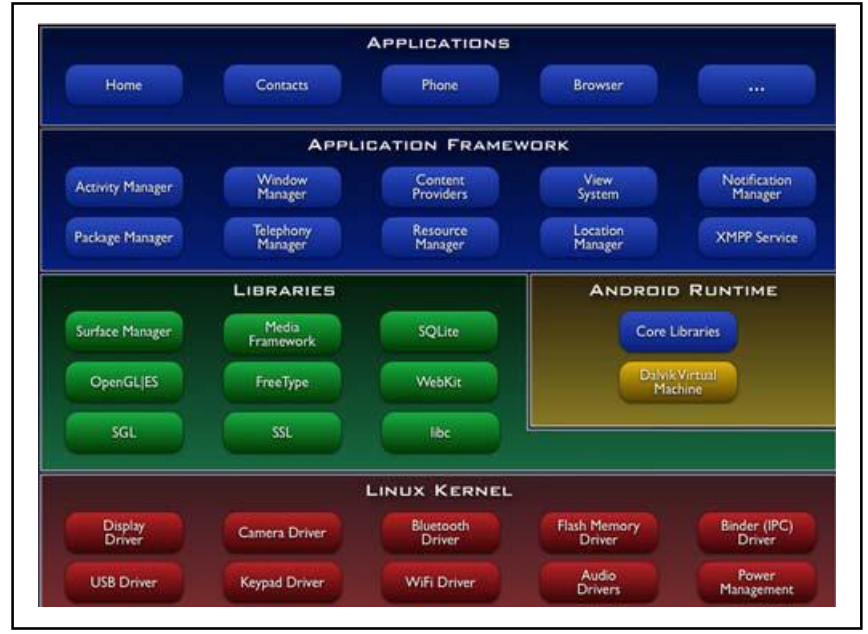

Figure 1. Android architecture

\section{A. Application}

On the top level of the framework is a set of core applications, released with Android, including an email client, a calendar, a SMS app, web browser, a map-application, contacts-app, etc. All Apps are written with the Java programming language.

\section{B. Application Framework}

Application Framework is the base of developing Apps in Android, most time the developers are working with Application Framework. The main components of Application Framework are: the activity manager, the window manager, content providers, the view system, the notification manager, the package manager, the telephony manager, the resource manager, the location manager and XMPP service [6][7][8].

In Android, every application can release its component and other applications can use the component released. The application architecture is designed to simplify the reusing of all components. This mechanism allows every component to be replaced by the user, it is impossible on Symbian and Windows Mobile.

\section{Libraries}

Android includes a set of $\mathrm{C} / \mathrm{C}++$ libraries used by various components of the Android system. The main core libraries are: libc, Media Framework, WebKit, SGL, OpenGL ES,

Supported by Chinese Universities Scientific Fund. 
FreeType, SQLite, etc. These capabilities are exposed to developers through the Android application framework.

\section{Android Runtime}

In Android, users develop applications with Java programming language, but Android doesn't use J2ME to run Java programs, it uses its own Android runtime. Android runtime includes a set of core libraries and the Dalvik virtual machine as shown in Fig. 2 [9].

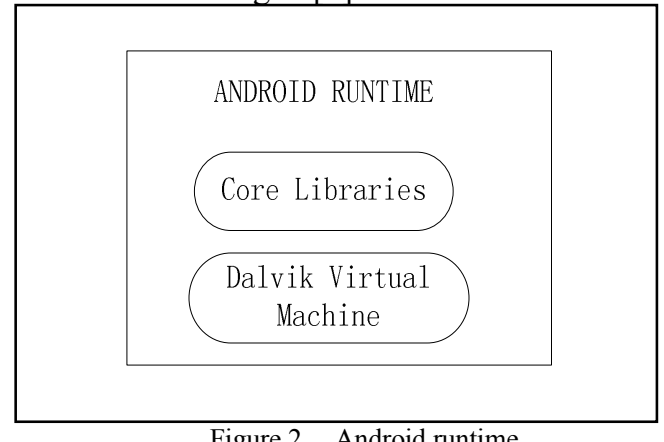

Figure 2. Android runtime

The core libraries include two parts. One provides most of the functionality available in the core libraries of the Java programming language. The other one is Android core libraries: such as android.os, android.net, android.media, etc. Every Android application runs in its own process given by the OS, and owns its own instance of the Dalvik virtual machine.

Dalvik is a register-based Java VM. The Dalvik VM is executing files in the dex (Dalvik Executable) format which was optimized for minimal cpu-and-memory-usage and supports running multiple VMs efficiently.

The Virtual Machine runs classes compiled by a Java language compiler that have been transformed at compile-time into the .dex format using the " $\mathrm{dx}$ " tools, that are shipped with the SDK.

\section{E. Linux Kernel}

Android relies on Linux (Kernel version 2.6) for core system services such as security, memory management, process management, network stack, and driver model. The Linux core also acts as a hardware abstraction layer between the applications and all the hardware, developers don't need to care about the hardware.

\section{Design Of Media Player}

\section{A. Design of media player based on hiberarchy}

To play media files, the media player should gather the media data first, decode the audio and video streams later, then display the data after decoding. According to the three steps, this paper design the media player based on hiberarchy. During the three steps, media player needs to parse the coding format of the media file, decode for the original data by the corresponding decode programs, put the original data to buffer queues, then display the original data after being synchronized.

This media player is based on hiberarchy, every layer completes its assignments by itself, this design reduces the application coupling. These layers from the top down are data extract layer, pretreatment layer, decode layer and the UI. The hiberarchy is shown in Fig. 3.

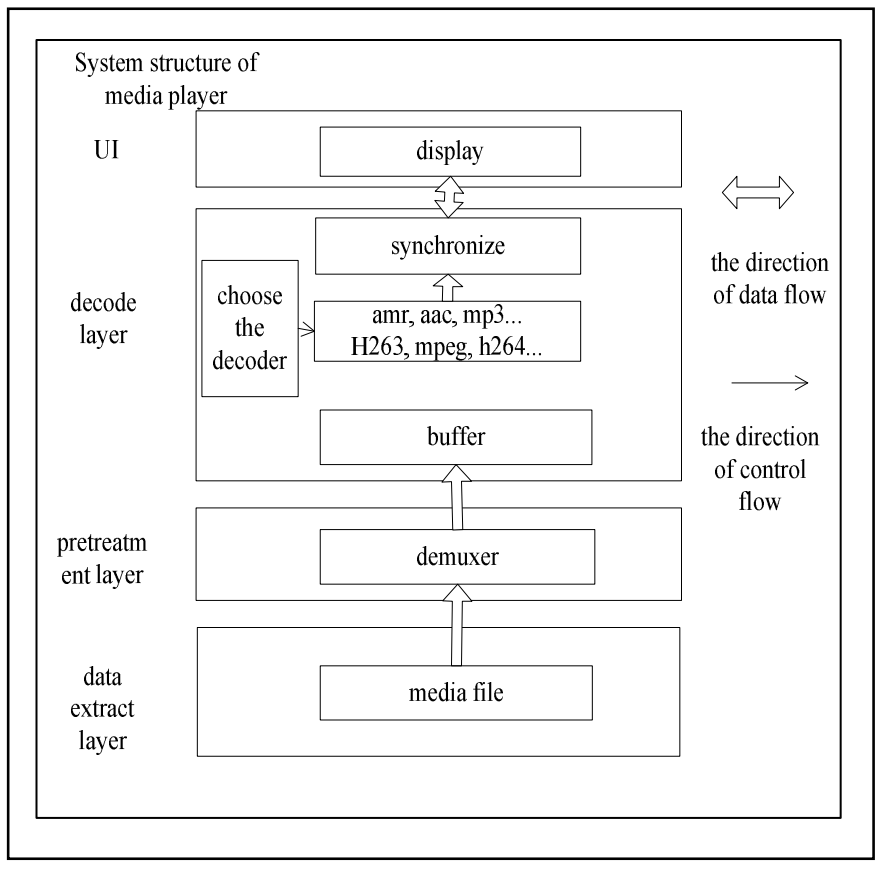

Figure 3. System structure of media player

Data extract layer is in charge of reading the media file. Pretreatment layer is to demux the media file and store the information of the media file into the buffer. Decode layer is to choose the corresponding decoder to decode audio streams and video streams, and then to synchronize the audio streams and the video streams. UI is to display the original data for users.

UI provides the interface for interaction between users and media player, such as functions of play, pause, page down page up, etc.

Decode layer includes the decoder choosing module, all kinds of decoders and the synchronize module. The decoder choosing module gathers the information of the media file's formats, decodes streams by the corresponding decoder. To separate decoders and the decoder choosing module is for the convenient of adding other kinds of decoders, in order to do so, users just need to register a new format decoder. The synchronize module is to synchronize audio streams and video streams before displaying a video.

Pretreatment layer is to demux the media file according to the format, and then put these streams into buffer.

Data extract layer is to read media files.

\section{B. Design of decode layer}

Decode layer is to separate decoders and the decoder choosing module. Every format decode unit in decoders is a module, before decoding, it is necessary to register all the formats which can be decoded by the module, then provide a link to connect a media format and the corresponding decode unit. The flow chat of this layer is shown in Fig. 4.

The structure reduces the coupling of this layer, meanwhile increases its expansibility. 


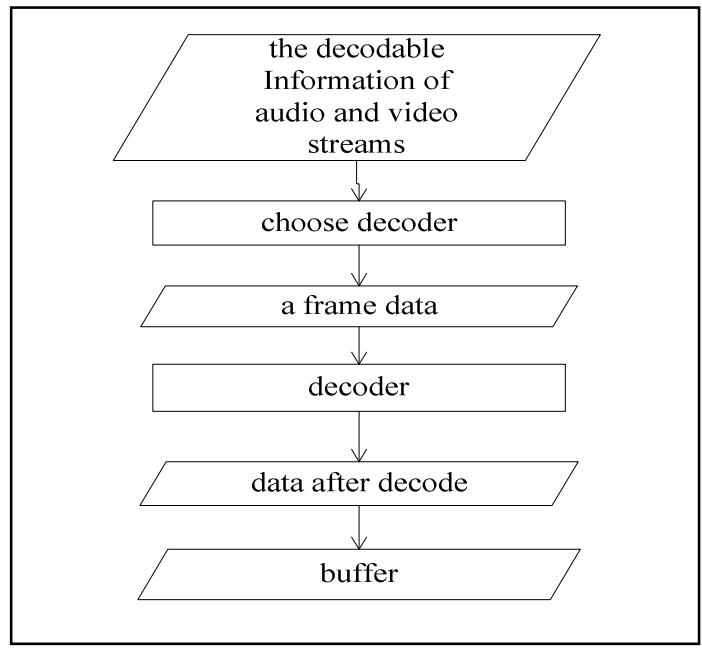

Figure 4. The flow chat of decode

\section{Design of decoder}

This paper designs decoder based on FFmpeg decode library.

The main flow of the decoder is shown in Fig. 5.

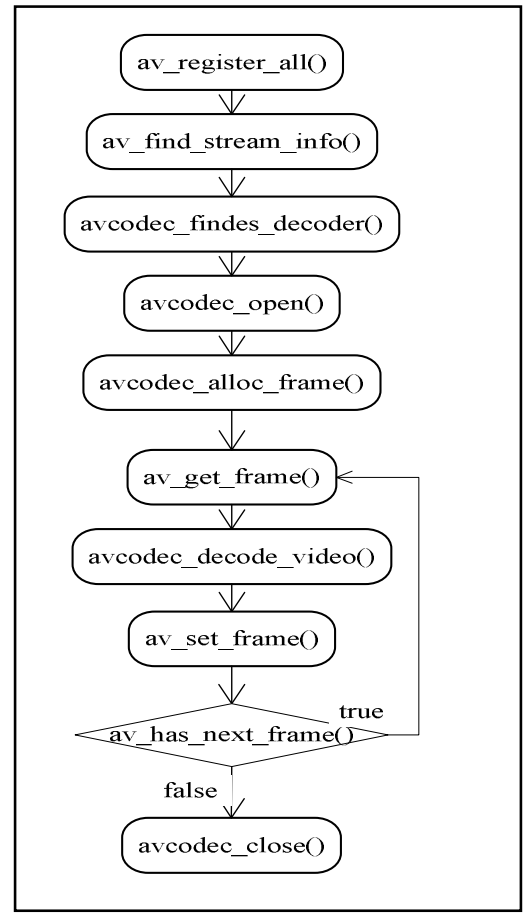

Figure 5. The flow chat of decoder

Every step's function is as follows:

1) av_register_all(): register all kinds of codec formats;

2) av find_stream_info(): get the decodable information from the media file;

3) avcodec_finds_decoder(): find the corresponding decoder;

4) avcodec_open(): open the decoder;

5) avcodec_alloc_frame(): allocate memory for decoding the frame;
6) av_get_frame(): get a frame data from the decode buffer:;

7) avcodec decode video(): decode the frame data;

8) av_set frame(): put the data into the display buffer;

9) If there are data for decoding then goto 6 step;

10) avcodec close(): after decoding, free the decoder;

The media player create a thread for every kind stream to execute these actions, a usual media file has two kind streams, audio streams and video streams, every thread has its own decode buffer and display buffer.

\section{IMPLEMENTATION OF MEdiA PlayeR}

\section{A. The transplantation of FFmpeg}

FFmpeg is based on Linux, now we must transplant it to Android. In Android, applications are developed with Java programming language based on Android SDK, but FFmpeg is based on $\mathrm{C}$ programming language. This paper develops dynamic linked library based $\mathrm{C}$ programming language (.so) by JNI, and then packs the .so file and the Java application as a .apk file by Android NDK. Firstly, because of phraseological difference we need to rewrite the makefile in FFmpeg, just like Android.mk; secondly, we compile and transplant avutil, avcodec, avformat orderly. The transplantation of FFmpeg has finished.

\section{B. Implementation of the play module}

The play module is to demux media files and to decode audio streams and video streams. This module is mainly based on FFmpeg. The transplantation is based on JNI, therefore media player invokes .so files in lowest-level by Java in top-level when decoding.

The invoke relation is shown in Fig. 6.

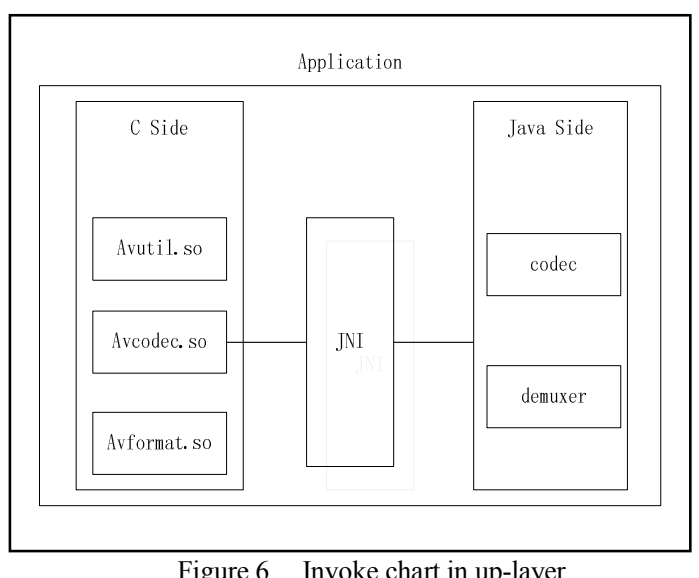

\section{Implementation of UI}

It is a complex project to display audio and video information. This paper uses open-source SDL library, where SDL (Simple DirectMedia Layer) is a cross-platform multimedia library designed to provide low level access to multimedia devices. This paper mainly uses video, audio, multithreading, timer, event and many other functions in SDL.

1) The main flow when playing the video streaming
a) Initial SDL library: 
SDL_Init(SDL_INIT_VIDEO | SDL_INIT_AUDIO SDL_INIT TIMER), the initialization of the SDL library requires for the video subsystem, audio subsystem and timer subsystem;

\section{b) Select and set the video mode:}

SDL_SetVideoMode(pCodecCtx->width, pCodecCtx ->height,0,0); the first two parameters are player window's width and height, the third parameter marked as 0 shows the use of the current pixel-bit color accuracy, and the fourth parameter confirms the layer structure;

c) Screen refresh function: schedule_refresh(is, 40);

\section{2) Playing control}

When playing, the user controls the video through the touch screen or the keyboard, such as pause, stop, and fast forward etc. The control part is SDL's SDL_WaitEvent() function. Part of the keyboard code is as follows.

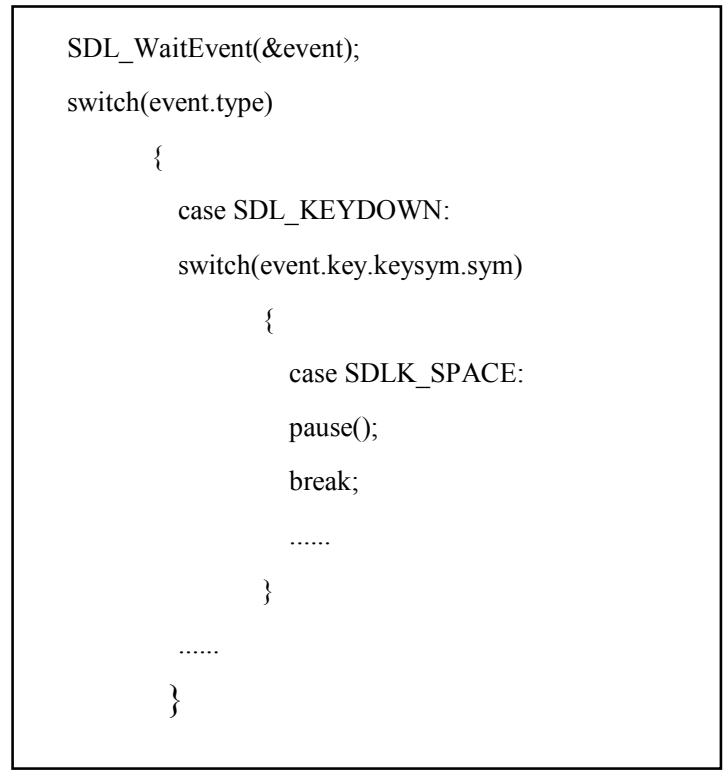

The play interface is shown in Fig. 7.

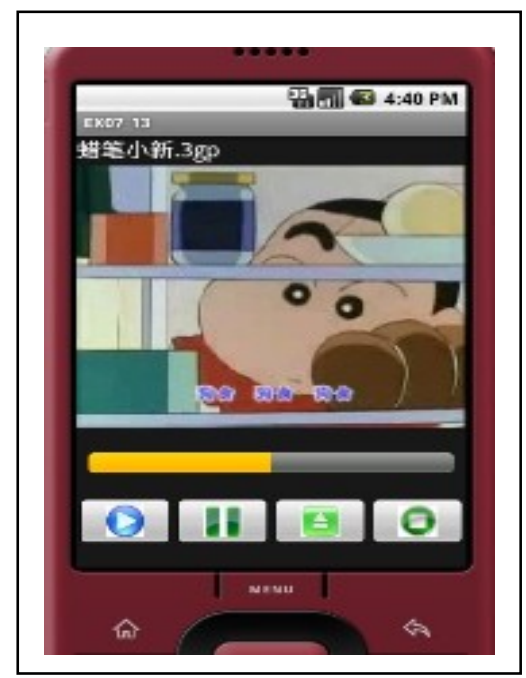

Figure 7. Play interface

\section{CONCLUSION}

After in-depth study of Android platform and FFmpeg, the paper designs and implements a powerful media player based on Android. In the paper the decode module is based on FFmpeg decode library; the display module is based on SDL; the structure of media player is based on hiberarchy, this structure reduces the coupling of this layer, meanwhile increases its expansibility; the transplantation of FFmpeg is mainly based on JNI invoke by Android SDK. Compare to the original media player in Android, the media player in this paper supports more media formats, the decode efficiency is higher.

\section{REFERENCES}

[1] Open Handset Alliance, Open Handset Alliance Announces 14 New Members, http://www.openhandsetalliance.com/press_120908.html [last accessed on Mar 1, 2009].

[2] Android home page, at http://www.android.com/. last visited on April 22, 2009.

[3] http://FFmpeg.mplayerhq.hu/index.html, "FFmpeg Multimedia System".

[4] J.F. DiMarzio, Android A Programmer's Guide, Chicago: McGraw-Hill, Jul. 2008.

[5] Android Developers, http://www.androidin.com/.

[6] Developer resources for Google Android: http://developer.android.com/.

[7] C. Haseman, Android Essentials, PDF Electronic Book, 2008. Available from: http://androidos.cc/dev/index.php.

[8] N. Gramlich, Android Programming , PDF Electronic Book, 2008. Available from: http://androidos.cc/dev/index.php.

[9] Android - An Open Handset Alliance Project: http://code.google.com/intl/zh-CN/android/. 